\title{
Hans-Wilhelm Eckert
}

Konservative Revolution in Frankreich? 


\section{Studien zur Zeitgeschichte}

Herausgegeben vom Institut für Zeitgeschichte

Band 58

R. Oldenbourg Verlag München 2000 


\section{Hans-Wilhelm Eckert}

\section{Konservative Revolution \\ in Frankreich?}

Die Nonkonformisten der Jeune Droite und des Ordre Nouveau in der Krise der 30er Jahre 
Die Deutsche Bibliothek - CIP-Einheitsaufnahme

\section{Eckert, Hans-Wilhelm:}

Konservative Revolution in Frankreich? : die Nonkonformisten

der Jeune Droite und des Ordre Nouveau in der Krise der 30er Jahre /

Hans-Wilhelm Eckert. - München : Oldenbourg, 2000

(Studien zur Zeitgeschichte ; Bd. 58)

Zugl.: Trier, Univ., Diss., 1996

ISBN 3-486-56441-2

(C) 2000 Oldenbourg Wissenschaftsverlag $\mathrm{GmbH}$, München Rosenheimer Straße 145, D-81671 München

Internet: http://www.oldenbourg-verlag.de

Das Werk einschließlich aller Abbildungen ist urheberrechtlich geschützt. Jede Verwertung außerhalb der Grenzen des Urheberrechtsgesetzes ist ohne Zustimmung des Verlages unzulässig und strafbar. Dies gilt insbesondere für Vervielfältigungen, Übersetzungen, Mikroverfilmungen und die Einspeicherung und Bearbeitung in elektronischen Systemen.

Umschlaggestaltung: Dieter Vollendorf

Gedruckt auf säurefreiem, alterungsbeständigem Papier (chlorfrei gebleicht).

Gesamtherstellung: R. Oldenbourg Graphische Betriebe Druckerei GmbH, München 


\section{Inhalt}

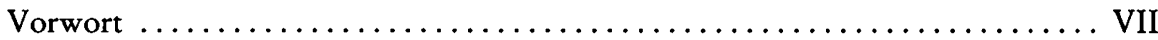

Einleitung $\ldots \ldots \ldots \ldots \ldots \ldots \ldots \ldots \ldots \ldots \ldots \ldots \ldots \ldots \ldots \ldots \ldots, 1$

I. Krise und Kritik: Zur Welle des antidemokratischen Denkens in der

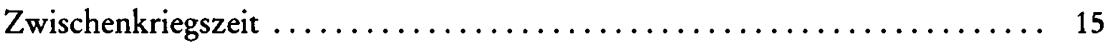

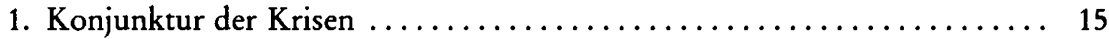

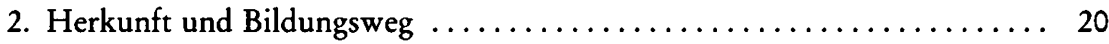

3. Generationserfahrungen und Jugendkult $\ldots \ldots \ldots \ldots \ldots \ldots \ldots \ldots \ldots \ldots$

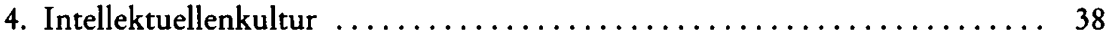

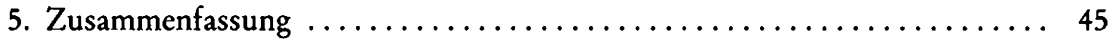

II. Aufbruch und Annäherung: 1930 bis $1933 \ldots \ldots \ldots \ldots \ldots \ldots \ldots \ldots \ldots$

1. Die Anfänge der Jeune Droite . . . . . . . . . . . . . . 49

Die Krise der Action française (49) - Jean de Fabrègues und Réaction (53) -

Jean-Pierre Maxence und die Revue française (57) - Revue du siècle und Revue $d u X X^{e}$ siècle (61)

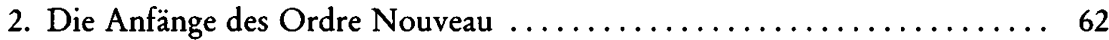

Der Club du Moulin Vert (62) - Plans (71) - Mouvements (74) - Esprit (76) -

Ordre Nouveau (78)

3. Auf dem Weg zu einer Dritten Front ................... 80

Erste Annäherungen (80) - Cahier de Revendications (84) - Union pour la vérité (86)

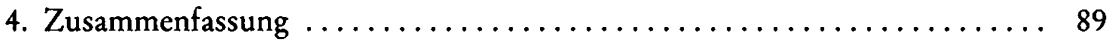

III. „Europa in Gefahr“: Alte Feinde - neue Verbündete .............. 91

1. Innere und äußere Bedrohung $\ldots \ldots \ldots \ldots \ldots \ldots \ldots \ldots \ldots \ldots \ldots \ldots \ldots \ldots$

Antibriandismus (93) - Antiamerikanismus (98)

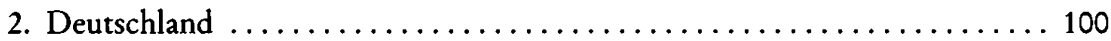

Die Weimarer Republik (100) - Der Nationalsozialismus (113)

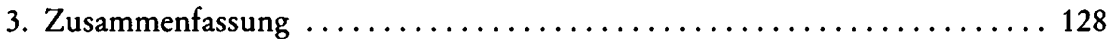

IV. Neue Bündnisse: Februar 1934 bis Oktober $1935 \ldots \ldots \ldots \ldots \ldots \ldots \ldots \ldots 131$

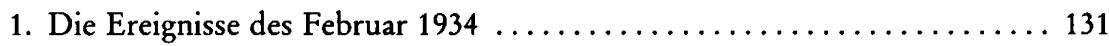

2. Isolation oder Aufbruch? ......................... 132

3. Antikapitalismus und Rassemblement-Idee ................. 136

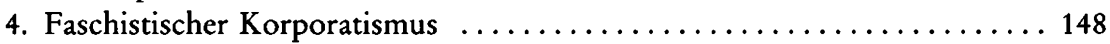

5. Die Sammlungsbewegung der Dissidenten $\ldots \ldots \ldots \ldots \ldots \ldots \ldots \ldots \ldots \ldots$

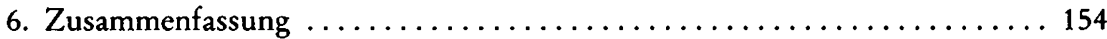

V. Jahre der Entscheidung: Ende 1935 bis $1939 \ldots \ldots \ldots \ldots \ldots \ldots \ldots \ldots \ldots \ldots$

1. Radikalisierung und Neopazifismus . . . . . . . . . . . . . 157

Der Abessinienkrieg (158) - Der französisch-russische Beistandspakt (163) -

Die Remilitarisierung des Rheinlands (167) 
2. Revolutionärer Nationalismus: Jeune Droite $\ldots \ldots \ldots \ldots \ldots \ldots \ldots \ldots$ Combat (170) - Comité National de Vigilance (173) - Insurgé - Sprachrohr der Cagoule? (176)

3. Nationaler Sozialismus: Ordre Nouveau

Zwischen den Fronten (184) - Orientierung zur Linken (190)

4. Antisemitismus und Antiliberalismus ........................ 192

5. Das Ende der Volksfront ............................ 208

6. Zwischen Neopazifismus und Machtpolitik .................. 211

Der ,Anschluß` Österreichs (211) - Das Münchener Abkommen (214) -

Der Einmarsch in Prag (218)

7. Zusammenfassung

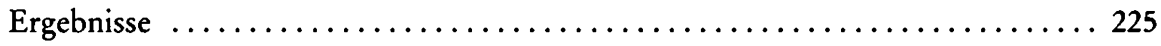

Abkürzungen ......................................... 237

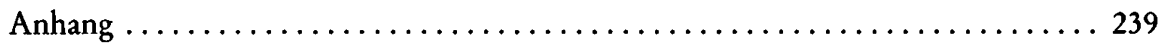

Kurzbiographien ................................... 239

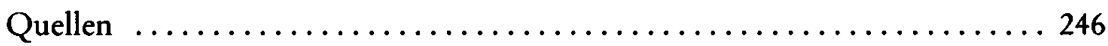

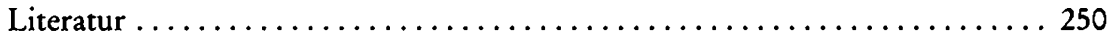

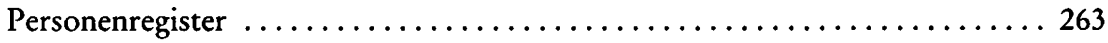




\section{Vorwort}

Die erste Gelegenheit zur Auseinandersetzung mit den französischen Intellektuellen bot sich für mich 1989 in Poitiers, wo ich im Rahmen eines Erasmus-Programmes ein Studienjahr verbringen durfte. In den Lehrveranstaltungen von Professor Jacques Valette erhielt ich wesentliche Einblicke in die Geschichte der sogenannten Nonkonformisten. Er regte mich dazu an, mich eingehender mit dem Thema zu beschäftigen.

Zurück in Deutschland unterstützte und förderte mein akademischer Lehrer Professor Wolfram Siemann meine Forschungen. Von ihm erhielt ich wichtige Anmerkungen zum methodischen Vorgehen. Sein kritisches Hinterfragen ermutigte mich, die Fragestellung auf eine Sozialgeschichte der Ideen zu konzentrieren.

Das vorliegende Buch ist die überarbeitete Fassung meiner Dissertation, die ich 1996 im Fachbereich Geschichte an der Universität Trier eingereicht habe. Dort wurden meine Forschungen von 1993 bis 1996 am Graduiertenkolleg "Westeuropa in vergleichender historischer Perspektive" aus den Mitteln der DFG gefördert.

Mit freundschaftlichem Dank sei an dieser Stelle besonders Andreas Wirsching erwähnt. Die im Buch vertretenen Thesen nahmen in zahlreichen Gesprächen mit ihm Kontur an. Er half mir, die Intellektuellengruppierungen in den Diskussionszusammenhang von Faschismus, Rechtsextremismus und Antiliberalismus einzuordnen. Ebenso bin ich Bruno Ackermann und Hans Coppi für ihre Unterstützung bei der Materialsuche und für den anregenden fachlichen Austausch zu Dank verpflichtet.

Alexandre Marc, einer der letzten Zeitzeugen der von mir untersuchten Kreise, gab mir in einem ausführlichen Gespräch wertvolle Einblicke in die Pariser Intellektuellenzirkel der dreißiger Jahre. Ihm sei an dieser Stelle sehr herzlich gedankt für sein Vertrauen und sein Entgegenkommen bei der Unterstützung meiner Recherchen.

Besonders erwähnen möchte ich die Mitarbeiter der Bibliotheken und Archive, deren freundliche Unterstützung und Hilfsbereitschaft meine Recherchen erheblich erleichtert haben. Insbesondere bedanke ich mich bei den Mitarbeitern der Staatsbibliothek München, der Archives Denis de Rougemont in Neuchâtel, der Bibliotheque Nationale und der Archives Nationales in Paris.

Den Herausgebern der Studien zur Zeitgeschichte sei für die Aufnahme meines Buches in ihre Reihe gedankt. Ihrer Lektorin, Petra Weber, danke ich für die Ausdauer, mit der sie geholfen hat, das Manuskript inhaltlich und stilistisch zu verbessern. 
Für Andrea und Jan 


\section{Einleitung}

Bei dem Begriff der „Konservativen Revolution“ handelt es sich um eine der „erfolgreichsten Schöpfungen der neueren Ideengeschichtsschreibung", wie Stefan Breuer feststellt ${ }^{1}$. Daran ändert auch die Tatsache nichts, daß der Begriff mehr Verwirrung stiftet als Klarheit schafft. Als Sammelbegriff für die neue Rechte in der Weimarer Republik hat er sich etabliert. Er umfaßt die antiliberalen Ideen einer ganzen Reihe von Persönlichkeiten und Gruppierungen, die in ihrer Radikalität über die traditionelle Rechte der Deutschnationalen hinauswiesen, die sich andererseits aber mehr oder weniger deutlich von den Nationalsozialisten abgrenzten. $\mathrm{Zu}$ ihren einflußreichsten Persönlichkeiten zählten Ernst Jünger, Carl Schmitt und Arthur Moeller van den Bruck.

Die Frage, ob es in Frankreich ein vergleichbares Phänomen gab, setzt voraus, daß es sich bei der Konservativen Revolution nicht um eine spezifisch deutsche Erscheinung handelte. Genau darin ist die Forschung häufig dem Selbstverständnis der Protagonisten gefolgt und hat dies mehr oder weniger stillschweigend unterstellt. Die Ideen der "politischen Romantik" 2 wurden zum geistigen Bezugspunkt eines spezifisch deutschen Irrationalismus erklärt ${ }^{3}$. Die Konservative Revolution stellt nach Ansicht von Martin Greiffenhagen "die letzte Phase eines spezifisch deutschen Konservatismus" dar ${ }^{4}$. Allerdings reicht die nationale Perspektive kaum aus, um die Besonderheit des Phänomens zu erkennen. Denn die Ablehnung des Liberalismus in der Zwischenkriegszeit blieb keineswegs auf Deutschland beschränkt, sondern erfaßte in unterschiedlicher Weise alle Länder Europas.

Erst in den letzten Jahren wurde die isolierte Betrachtungsweise überhaupt als Problem erkannt. Stefan Breuer und Rolf Peter Sieferle 5 betonen in ihren Arbeiten zur Konservativen Revolution ausdrücklich, daß es sich keineswegs um ein ausschließlich deutsches Phänomen handle. Breuer beklagt zu Recht, daß die bisherigen Untersuchungen trotz gegenteiliger Beteuerungen immer „zutiefst germanozentrisch “6 geprägt waren, und versteht seine Analyse als Ausgangspunkt für eine vergleichende Perspektive. Sie-

1 Breuer, Anatomie, S. 1.

2 So der Titel eines Werkes von Carl Schmitt 1919.

${ }^{3}$ Vgl. Sontheimer, Antidemokratisches Denken, S. 20; Fritzsche, Politische Romantik. Hans Mommsen schreibt über die Konzeptionen eines „Dritten Weges“, wie sie in der Konservativen Revolution vertreten wurden: „All diesen Konzeptionen war gemeinsam, daß sie von den sozialen und ökonomischen Bedingungen der Industriegesellschaft weithin abstrahierten und ganz im Sinne der deutschen politischen Tradition und unter dem Einfluß neoromantischer Ideen eine konfliktfreie Gesellschaft postulierten." Mommsen, Generationenkonflikt, S. 56.

${ }^{4}$ Greiffenhagen, Dilemma, S. 243. Ansätze zu einer vergleichenden Perspektive, wie sie Stern und Mosse entwickelt haben, wurden in der deutschen Historiographie nicht weiter verfolgt. Allerdings konzentrieren sich diese Untersuchungen jeweils nur auf einen Teilaspekt, den Kulturpessimismus (Stern) und den Korporatismus (Mosse), von dem aus die Besonderheit der Konservativen Revolution nicht erfaßt werden kann. Stern, Politics of Cultural Despair; Mosse, Jews.

${ }^{5}$ Konservative Revolution.

${ }^{6}$ Breuer, Anatomie, S. 182. Der Vorwurf fällt allerdings auf ihn zurück, da er selbst die einschlägigen und in diesem Fall besonders aufschlußreichen Untersuchungen zu Frankreich nicht kennt. 
ferle versucht einleitend, den geistigen Ursprung der Konservativen Revolution innerhalb einer gesamteuropäischen Gegenbewegung zu Liberalismus und Sozialismus zu lokalisieren?.

Mit diesen Analysen sind Anknüpfungspunkte geschaffen, die einen Brückenschlag zu den antiliberalen Gruppierungen in Frankreich ermöglichen. Ein Blick über den Rhein zeigt, daß auch dort eine Reihe von Intellektuellen die Grundlagen des parlamentarischen Systems attackierte, den Niedergang der eigenen Nation diagnostizierte und sich nach einer radikalen Erneuerung sehnte. Zu diesen Protagonisten gehörten die sogenannten Nonkonformisten, die zu Beginn der dreißiger Jahre von sich reden machten. Zwei der bedeutendsten Gruppierungen aus diesem Kreis, die Jeune Droite und der Ordre Nouveau, bilden den empirischen Schwerpunkt dieser Untersuchung. Die vergleichende Perspektive soll Parallelen zur Konservativen Revolution in Deutschland aufzeigen, um auf diese Weise Gemeinsamkeiten und Besonderheiten der Phänomene in den beiden Nachbarländern überhaupt erst sichtbar zu machen.

Ein Blick auf die Forschungslage in Frankreich zeigt einen ähnlichen Befund wie in Deutschland. Breuers Kritik an der Konzentration auf die nationale Perspektive läßt sich ebensogut auf die Kollegen in Frankreich übertragen. In den Untersuchungen zu den Nonkonformisten und zur französischen Rechten wurden die Entwicklungen im deutschen Nachbarland ebenfalls nicht wahrgenommen ${ }^{8}$. Erst in dem jüngst erschienenen Sammelband Ni gauche, ni droite wird der Versuch unternommen, diese nationalen Perspektiven zu durchbrechen und Anknüpfungspunkte für den Vergleich zu schaffen. Hans Manfred Bock hat auf Parallelen und Verbindungen zwischen der Konservativen Revolution und den Nonkonformisten hingewiesen'. Als zentrale Merkmale der Ideologien beiderseits des Rheins hebt Bock den radikalen Antiliberalismus und den revolutionären Nationalismus hervor. Sie zeigen eine ähnliche Sympathie für autoritäre Herrschaftskonzeptionen. Darüber hinaus stellt er vergleichbare Entwicklungen fest: Charakteristisch für die Konservative Revolution und den Nonkonformismus ist das Bemühen, traditionelle Parteistandpunkte zu überwinden und einen „Dritten Weg“ zwischen Kommunismus und Liberalismus zu beschreiten. Nicht zuletzt beeinflußten sich Konservative Revolution und Nonkonformismus wechselseitig durch die Rezeption der Ideen und durch persönliche Kontakte ${ }^{10}$.

\footnotetext{
7 Sieferle, Konservative Revolution, S. $7 \mathrm{ff}$.

${ }^{8}$ Loubet del Bayle, Non-conformistes; Sternhell, Ni droite; Rémond, Droites. Nicht einmal die französischen Kenner der Konservativen Revolution in Deutschland, Dupeux und Moreau, haben auf vergleichbare Phänomene in ihrem Land hingewiesen; vgl. Dupeux, „Nationalbolschewismus"; ders., „Kulturpessimismus“, S. 287-299; Moreau, Nationalsozialismus.

${ }^{9}$ Bock, Crise, S. 299-311. Der Sammelband ist aus einem Kongreß in Bordeaux 1991 hervorgegangen. Einen Zusammenhang zwischen der Konservativen Revolution und dem Denken des Ordre Nouveau hat bereits Pascal Sigoda skizziert. Er betrachtet die französische Gruppierung als Bindeglied zwischen dem Denken der Weimarer Rechten und dem General Charles de Gaulles. Seine Beweisführung beschränkt sich allerdings weitgehend auf die Rekonstruktion der Kontakte; Sigoda, de Gaulle, in: Espoir 46 (1984), S. 43-49.

10 Bock, Crise, S. 302ff.; vgl. Merlio, Préface, ebenda, S. 7-13. Die übrigen Beiträge konzentrieren sich auf nationale Phänomene, oder sie widmen sich der Rezeptionsgeschichte und den persönlichen Beziehungen.
} 
Damit hat Bock die unterschiedlichen Ebenen skizziert, auf denen die vergleichende Perspektive entwickelt werden muß. Bevor jedoch Übereinstimmungen in den Entwicklungen und direkte Einflüsse untersucht werden können, müssen in einem ersten Schritt die Parallelen auf der ideologischen Ebene genauer bestimmt werden. Zu diesem Zweck werde ich die vorliegenden Untersuchungen zur Konservativen Revolution und zum Nonkonformismus vorstellen und auf einen gemeinsamen begrifflichen Kernbestand hin untersuchen.

Den Begriff der „Konservativen Revolution“ hat Armin Mohler 1949 mit seiner von Karl Jaspers und Hermann Schmalenbach betreuten Dissertation in die wissenschaftliche Diskussion eingeführt. Er betrachtet das Phänomen als die radikalste Antwort auf die Ideen der Französischen Revolution ${ }^{11}$. Einen ideologischen Kernbestand sieht Mohler in der von Nietzsche formulierten Denkfigur der „ewigen Wiederkehr des immer Gleichen “. Sie richte sich gegen alle linearen Geschichtsbilder: gegen den Erlösungsglauben des Christentums ebenso wie gegen die Fortschrittsidee der Aufklärung und gegen die Teleologie des Marxismus. Nicht eine klar umrissene Ideologie, sondern eine vorbewußte Haltung oder „Weltanschauung “ bildet demnach den zentralen Kern dieses Denkens $^{12}$.

Die Diagnose einer radikalen Feindschaft zur Moderne (wenn man diese mit dem ausgehenden 18. Jahrhundert beginnen läßt) hat den Blick darauf verstellt, daß die Protagonisten der Konservativen Revolution in eben dieser Moderne verwurzelt waren, die sie so heftig bekämpften. Panajotis Kondylis hat erstmals darauf hingewiesen, daß der Konservativismus seinen Bezugspunkt bereits verloren hatte, als sich die Konservativen Revolutionäre seiner Versatzstücke bedienten. Kondylis zufolge läßt sich im „Konservativismus" nur dann ein konkreter Gehalt ausmachen, wenn man ihn einer bestimmten Epoche und einem sozialen Träger zuordnet. Der Konservativismus war seiner Ansicht nach die Legitimationsideologie des Adels, der das alteuropäische Gesellschaftsideal der societas civilis bewahren wollte. Diese Ordnung hatte seit dem Beginn der Neuzeit vor allem zwei Gegner: den absolutistischen Staat und das Bürgertum. Autonomie und Herrschaft des adligen Hauses wurden durch die Entwicklung von Märkten und Handelsbeziehungen in Frage gestellt, die Machtstellung des Adels wurde durch die Zentralisierung politischer Entscheidungen im absolutistischen Staat bedroht. Der Konservativismus richte sich daher sowohl gegen den bürgerlichen Kapitalismus als auch gegen die moderne Souveränitätslehre ${ }^{13}$.

Mit der Französischen Revolution aber war diese Ideologie ihres Bezugspunktes weitgehend beraubt. Der Konservativismus nach 1789 läßt sich Kondylis zufolge nur noch als eine Geschichte fortdauernder Rückzugsgefechte beschreiben. Feudalistische Überreste waren in Deutschland mit der Bauernbefreiung und der Aufhebung der Patri-

${ }^{11}$ Inzwischen liegt die vierte Auflage vor, ergänzt durch einen umfassenden bibliographischen Anhang: Mohler, Konservative Revolution, Bd. 1, S. 10f. Diese Feindschaft zur Französischen Revolution verbindet die Konservative Revolution nach Ansicht Mohlers mit den reaktionären, altkonservativen und restaurativen Kräften, die üblicherweise unter dem Begriff „konservativ“ $z$ usammengefaßt werden. Vgl. Vierhaus, Konservativ, S. 531-565; Mannheim, Konservatismus; Valjavec, Entstehung, S. 255ff.; Greiffenhagen, Dilemma, S. 27ff.

12 Mohler, Konservative Revolution, Bd. 1, S. 88.

${ }^{13}$ Kondylis, Konservativismus, S. $22 \mathrm{ff}$. 
monialgerichtsbarkeit bis 1848 weitgehend beseitigt, auch wenn der Adel hier noch bis zum Ersten Weltkrieg führende Positionen in Armee und Politik innehatte. Um ihre Stellung zu behaupten, mußten die Konservativen zentrale Leitbegriffe ihres Denkens wie die Ablehnung des gesatzten Rechts und die damit verbundene Absage an die moderne Souveränitätslehre aufgeben. Sie haben damit nolens volens die moderne Staatlichkeit akzeptiert und sogar den Nationalismus übernommen. Wenn die Bezeichnung „konservativ“ weiterhin gebraucht wurde, so darf dies nach Ansicht von Kondylis nicht darüber hinwegtäuschen, daß eine ideologische Kontinuität unter dem Deckmantel des Wortes nur vorgetäuscht wurde. Eine substantielle Trennung von Liberalismus und Konservativismus war gegen Ende des 19. Jahrhunderts unmöglich geworden ${ }^{14}$.

An diese Überlegungen knüpft Stefan Breuer an. Für ihn handelt es sich bei der Konservativen Revolution nicht um eine Reaktion gegen die Moderne, sondern innerhalb der Moderne, die sich gegen bestimmte Formen und Auswüchse derselben richte. Breuer siedelt das Phänomen in der Übergangsphase von der bürgerlichen Industriegesellschaft in eine industrielle Massengesellschaft an. Mit dem sozialen Wandel seien zugleich die geistigen Grundlagen der Moderne selbst ins Wanken geraten. Die Vernunft als Instrument der Emanzipation und der Bekämpfung ständischer Privilegien wurde nun ihrerseits zum Problem. Der rationalistische Individualismus habe sich in zunehmendem Maße als ungeeignet erwiesen, die Gesetze und Kräfte sozialer Entwicklungen $\mathrm{zu}$ erklären und zu beherrschen ${ }^{15}$.

Ein starkes voluntaristisches Element prägte die Herrschaftsentwürfe seit dem Ende des 19. Jahrhunderts. Die neuen ästhetischen und moralischen Wertvorstellungen bezogen ihre Legitimation nicht mehr aus der Anknüpfung an traditionelle Herrschaftsformen. Diese spielten nur noch in idealisierter Form, etwa als rückwärtsgewandte Kapitalismuskritik, eine Rolle. Auch die Elitekonzeptionen wandelten sich: An die Stelle des Adels traten Künstler, Industrielle und charismatische Herrscher ${ }^{16}$. Ein Beispiel für diesen Voluntarismus liefert Arthur Moeller van den Bruck, der 1923 die Überwindung der Weimarer Republik durch ein Drittes Reich prophezeite, das die Konservativen wieder an die Macht bringe. Konservativ zu sein bedeutete für ihn, „Dinge zu erschaffen, die zu erhalten sich lohnt ${ }^{{ }^{17}}$. Auch Rolf Peter Sieferle siedelt den geistigen Ursprung der Konservativen Revolution in der Umbruchsituation am Ende des 19. Jahrhunderts an. Im Unterschied zu Breuer und Kondylis betont er, daß es sich dabei um ein Phänomen handle, das von beiden Rändern des politischen Spektrums bestimmt war. Die extreme Rechte und die extreme Linke gingen seiner Ansicht nach von den gleichen kulturellweltanschaulichen Grundlagen aus. Die Beziehungen zwischen beiden ließen sich daher keineswegs auf das Verhältnis von Aktion und Reaktion festlegen. Es handle sich vielmehr um konkurrierende Strömungen, die vor allem eines gemeinsam hatten: Es ging um die Erschaffung einer neuen Gesellschaft mit neuen Wertvorstellungen. Die jakobinischen Ursprünge dieser Entwürfe bestimmten ihren tendenziell totalitären Charakter. An die Stelle des vernünftigen Individuums seien Kollektivkräfte wie das Volk, die Na-

${ }^{14}$ Ebenda, S. $207 \mathrm{ff}$.

${ }^{15}$ Breuer, Anatomie, S. 15ff.; vgl. Beck, Risikogesellschaft, S. $14 \mathrm{f}$.

${ }^{16}$ Kondylis, Konservativismus, S. 447ff.

${ }^{17}$ Moeller van den Bruck, Das Dritte Reich, S. 215. Zu Moeller vgl. Goeldel, Moeller van den Bruck. 
tion, die Klasse, die Rasse getreten. Aus der Sicht beider Extrempole sei der Liberalismus in der Form der parlamentarischen Demokratie, des Kapitalismus und der bürgerlichen Gesellschaft nur ein „historisches Übergangsphänomen“ ${ }^{18}$ gewesen. Die Auseinandersetzung zwischen den extremen Flügeln der Rechten und der Linken wurde zu einem Wettkampf konkurrierender Projekte einer anderen Moderne - ein Wettkampf, der insbesondere durch die fundamentalen Erschütterungen des Weltkriegs eine neue Dimension gewonnen habe ${ }^{19}$.

An die Stelle einer dualistischen Einteilung des politischen Spektrums, die seit der Französischen Revolution üblich geworden war, sei somit ein triadisches Modell getreten. Dem Liberalismus stünden zwei neue, radikale Strömungen gegenüber: der marxistische Sozialismus und die Konservative Revolution ${ }^{20}$. Sieferle schlägt damit eine Maximaldefinition des Begriffes vor. Ebenso wie er den Bolschewismus als die Umsetzung des Marxismus betrachtet, erscheint auf der anderen Seite der Nationalsozialismus als "reale Konservative Revolution“21. Er fordert kein konsistentes theoretisches Programm, sondern die Möglichkeit eindeutiger Abgrenzung vom jeweiligen Gegner. Für Sieferle reicht es aus, daß sich von jeder der drei Grundpositionen die jeweils anderen beiden klar abgrenzen lassen und sie somit ihre polemische Funktion, die eindeutige Identifikation des Gegners, erfüllen. Aus der Perspektive des Liberalismus seien Konservative Revolution und Sozialismus Formen totalitärer Herrschaft, aus der Sicht des Sozialismus Liberalismus und Konservative Revolution Ausdruck bürgerlicher Herrschaft, für die Konservative Revolution schließlich seien Liberalismus und Sozialismus Auflösungserscheinungen der nationalen Gemeinschaft ${ }^{22}$.

Für Breuer bildet dagegen die Konservative Revolution nur einen Teil dieser neuen radikalen Rechten. Er geht umgekehrt wie Sieferle vor und bietet gewissermaßen die Minimaldefinition des Phänomens, indem er nach dem gemeinsamen ideologischen Kernbestand fragt. Dieser läßt sich - darin gibt er wiederum Mohler recht - nicht in einer klar umrissenen Ideologie fassen. Zu groß waren die Divergenzen in Fragen der Wirtschaftspolitik, in den Ansichten zu Wissenschaft und Technik bis hin zu den konkreten Vorstellungen von Nation, Volk, Rasse, Herrschaft und Reich ${ }^{23}$. Eine einheitliche Denkfigur diagnostiziert Breuer dagegen im neuen Nationalismus. Damit greift er einen Ansatz auf, den Kurt Sontheimer in seiner Untersuchung über „Antidemokratisches Denken in der Weimarer Republik“ entwickelt hat. Die „politischen Ideen des deutschen Nationalismus" - so der Untertitel des Werkes - richteten sich mit aller Kraft gegen die liberale Demokratie der Weimarer Republik. In dieser Feindschaft sieht er den Kern und die entscheidende Motivation des neuen Nationalismus. Das Neue an diesen Ideen war ihr Irrationalismus, ihre Dynamik und die Feindschaft zum Wilhelminismus und seiner materialistischen Weltordnung. Dadurch grenzten sie sich von den restaurativen Ideen der Deutschnationalen und auch von völkischem Gedankengut ab. Sontheimers großes Verdienst ist es, das Kriegserlebnis als Ursprung dieser neuen radikalen Strömungen lokali-

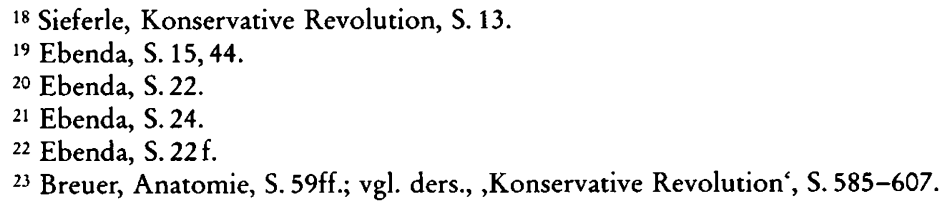


siert zu haben. In seiner Untersuchung zeigt er anschaulich, wie die geistige Mobilmachung und die kriegerischen Ideale in den Ideen der Konservativen Revolutionäre fortlebten. Für Sontheimer sind diese Ideen ein allerdings spezifisch deutsches Phänomen, das nur auf dem Nährboden des romantischen Irrationalismus und eines Ästhetizismus, wie er in den „Betrachtungen Unpolitischer" seinen Ausdruck gefunden hat, gedeihen konnte ${ }^{24}$.

Mit diesem Befund gibt sich Breuer nicht zufrieden. Er steht dieser Idee eines deutschen Sonderweges mit kritischer Distanz gegenüber. Auf der Suche nach einem gemeinsamen ideologischen Kernbestand arbeitet er vier Elemente des neuen Nationalismus heraus, die von der germanozentrischen Perspektive befreit sind und als Ansatzpunkt für eine vergleichende Perspektive dienen können. 1. Der neue Nationalismus war inklusiv, er wollte die Klassengegensätze ausdrücklich überwinden. 2. Er war revolutionär, er strebte den Sturz des Weimarer Systems an. 3. Er forderte einen autoritären, hierarchischen und sogar diktatorischen Staat. 4. Er besaß ein charismatisches Sendungsbewußtsein. Ähnlich wie der jakobinische Nationalismus hatte er den Charakter einer politischen Religion. Die Konservativen Revolutionäre waren seine Propheten ${ }^{25}$. Aus dieser Definition fallen die sogenannten Nationalbolschewisten heraus, weil sie nach Ansicht Breuers durch die Anerkennung des Klassenkampfes der extremen Linken zu nahestanden ${ }^{26}$. Ebenso überschreiten Oswald Spengler und Ernst Jünger in seinem 1932 erschienenen Essay „Der Arbeiter" den Rahmen, da sie ein weltumspannendes Imperium anvisierten, in dem die Nation zur obsoleten Größe geworden wäre ${ }^{27}$.

Die Welle des antiliberalen Protests in Frankreich hat Jean Touchard als „esprit des années trente" charakterisiert, mit dem sich eine neue Generation von Intellektuellen Gehör verschafft habe. Diese sogenannten Nonkonformisten zeichne vor allem eines aus: ihr radikaler Angriff auf das bestehende System. Er artikuliere sich auf mehreren Ebenen und richte sich gegen unterschiedliche Ausprägungen der bestehenden Ordnung: gegen die liberale Demokratie, gegen die kapitalistische Ordnung und gegen den Materialismus, der beiden zugrunde lag. In dieser negativen Einheit lassen sich die verschiedenen Gruppierungen am ehesten fassen. Größere Schwierigkeiten bereitet es der Forschung, einen gemeinsamen ideologischen Kernbestand zu finden.

Diese Welle des Protests markierte eine Zäsur im politischen Klima der Dritten Republik. Einerseits kündigte er das Ende der Nachkriegszeit an, die mit dem Young-Plan und der Rheinland-Räumung endgültig abgeschlossen war, andererseits war er der Auftakt zu jener Krise, die Frankreich während der gesamten dreißiger Jahre prägen sollte. Touchard beschreibt diesen „esprit“ als einen ersten Versuch, die Gräben zwischen den Anhängern verschiedener politischer Lager von den Sozialisten bis hin zu den Monar-

\footnotetext{
${ }^{24}$ Vgl. Sontheimer, Antidemokratisches Denken, S. $15 \mathrm{ff}$.

${ }^{25}$ Breuer, Anatomie, S. $186 f f$.

${ }^{26}$ Ebenda, S. 153.

${ }^{27}$ Ebenda, S. 113, 183. Breuer schlägt schließlich vor, den Begriff der Konservativen Revolution durch den des "Neuen Nationalismus" zu ersetzen. Es handelt sich jedoch dabei um eine bloße Frage der Terminologie. Niemand wird nach den Untersuchungen von Kondylis, Breuer und Sieferle daran festhalten, die Konservative Revolution für konservativ zu halten. Da sich aber der Begriff, wie Breuer selbst betont, als Forschungsbegriff etabliert hat, möchte ich im folgenden daran festhalten; vgl. Breuer, Anatomie, S. 182.
} 
chisten zu überwinden. Er sieht die Gemeinsamkeit der Intellektuellen weniger in ihren konkreten politischen Forderungen als vielmehr in dem radikalen Gestus, der Diagnose einer fundamentalen Krise, dem unbedingten Willen zum Bruch mit dem bestehenden System und dem Vokabular der Erneuerung ${ }^{28}$. Diesem Ansatz folgte auch Jean-Louis Loubet del Bayle, der in seiner großangelegten Untersuchung über die nonkonformistischen Kreise der Jeune Droite, des Ordre Nouveau und Esprit das bis heute maßgebliche Standardwerk dieser Intellektuellengeneration verfaßt hat ${ }^{29}$.

In einen neuen Zusammenhang hat der in Israel lehrende Historiker Zeev Sternhell die Nonkonformisten gerückt. In seinem 1983 erschienenen Werk $N i$ droite, ni gauche betrachtet er sie als die Protagonisten eines „fascisme spiritualiste“, die mit ihrer Revolte gegen den „Materialismus“ eine neue Synthese aus nationalen und sozialen Ideen formuliert haben ${ }^{30}$. Sternhell sieht im Faschismus ein primär ideologisches Phänomen, das sich gerade deshalb in Frankreich in Reinform habe entwickeln können, weil dort zumindest bis 1940 keine faschistische Bewegung an die Macht gekommen ist. Er beschreibt die Entstehung dieser Ideologie als die Suche nach einem „Dritten Weg“ zwischen Liberalismus und Sozialismus, als eine Reihe von Revisionsversuchen, die auf eine Integration der Massen in die nationale Gemeinschaft zielen. Von besonderer Bedeutung ist dabei, daß Sternhell das Auftreten der Nonkonformisten nicht als eine erste Revolte, sondern als den Höhepunkt einer geistigen Strömung wertet, die ihren Ursprung im ausgehenden 19. Jahrhundert hat. Seine These geht auf zwei Vorstudien über Maurice Barrès und die Entstehung der revolutionären Rechten seit 1885 zurück $^{31}$. In der Epoche, die Gustave Le Bon als „Ära der Massen“"32 beschrieb, lokalisiert er die geistigen Wurzeln des „Faschismus“. Er sieht in dem Phänomen eine Antwort auf die Krise des Liberalismus mit ihren Begleiterscheinungen, der Verstädterung und dem Auftreten eines militanten Industrieproletariats.

In dieser Situation formierte sich eine neue radikale Rechte, die sich an ihrem Gegenspieler auf der extremen Linken orientierte: sie mobilisierte die Massen und propagierte einen Kult der Gewalt und des Irrationalismus, der auf eine Revolution hinauslaufen und die liberale Ordnung zerstören sollte. Mit einer antikapitalistischen Programmatik appellierte sie an die städtischen Unterschichten. Ihr erklärtes Ziel war jedoch nicht die Herrschaft des Proletariats, sondern dessen Integration in die nationale Gemeinschaft ${ }^{33}$.

${ }^{28}$ Touchard, L'esprit, S. 89-120.

${ }^{29}$ Loubet del Bayle, Non-conformistes.

${ }^{30}$ Sternhell, Ni droite, S. 234ff. Diesen Ansatzpunkt hat er in einer jüngeren Untersuchung noch einmal bekräftigt und eine Kontinuitätslinie von Ernest Renan bis hin zu Esprit und Ordre Nouveau gezogen: Sternhell, Troisième voie, S. 17-29.

${ }^{31}$ Sternhell, Maurice Barrès; ders., Droite révolutionnaire.

${ }^{32}$ Le Bon, Psychologie, S. 3.

${ }^{33}$ Sternhell, Droite révolutionnaire, S. 26ff. Das Argumentationsmuster der Konservativen, sie besäßen im Unterschied zu ihrem Gegner kein kohärentes System, hat die neue Rechte übernommen. Dies ist jedoch nur eine taktische Schutzbehauptung, die belegen soll, daß die Ideologie der Rechten "organisch" gewachsen sei, während es sich bei der Ideologie der Linken um eine "abstrakte“ Konstruktion handele, die mit der Realität nichts zu tun habe. Allerdings mußte sich die Rechte bereits bei der Anerkennung eines Primats der Praxis über die Theorie auf dasselbe Abstraktionsniveau begeben wie ihr Gegner. Schließlich war der Herrschaftsanspruch der Rechten ebenso legitimationsbedürftig wie derjenige der Linken; vgl. Kondylis, Konservativismus, S. 16f., Sternhell, Droite révolutionnaire, S. 27. 
Die geistigen Väter dieser neuen Ideologie kamen jedoch nur zum Teil von der Rechten, wie Paul Déroulède, Jules Guérin, Gustave Le Bon, Maurice Barrès, Edouard Drumont und Charles Maurras. Sternhells besondere Aufmerksamkeit gilt den ehemaligen Linken, die sich vom orthodoxen Marxismus abgewendet haben und in das rechte Lager übergewechselt sind. Von ihnen gingen seiner Ansicht nach die zentralen Anregungen aus: die revolutionären Syndikalisten Georges Sorel und seine Schüler Edouard Berth und Hubert Lagardelle hatten nicht nur auf Mussolini einen maßgeblichen Einfluß, sondern auch auf die Action française und den Faisceau von Georges Valois ${ }^{34}$. In diesem Revisionismus von links und rechts, der in eine Synthese aus Nationalismus und Sozialismus mündete, sieht Sternhell den Kern der faschistischen Ideologie ${ }^{35}$.

Sternhells Thesen stießen bei den französischen Historikern vor allem aus zwei Gründen auf eine geschlossene Abwehrfront. Erstens betrachtet er den Faschismus als ein genuin französisches Phänomen, ohne das Mussolinis Bewegung überhaupt nicht denkbar gewesen wäre. Dagegen herrscht in der französischen Zeitgeschichtsschreibung die Ansicht vor, daß die Ähnlichkeiten zwischen den französischen Bewegungen und dem italienischen Faschismus rein äußerlicher Natur waren. Diese Interpretationslinie hat René Rémond in seiner großen Untersuchung über die französischen Rechten entwickelt. Die paramilitärischen Ligen in Frankreich standen seiner Ansicht nach in der Linie des plebiszitären Bonapartismus und waren daher weitgehend „immun“ gegen äußere Einflüsse. Der "Faschismus“ ist Rémond zufolge ein gänzlich unfranzösisches Phänomen ${ }^{36}$. Zweitens vertritt Sternhell die Auffassung, daß die faschistische Ideologie in Frankreich in den dreißiger Jahren nahezu omnipräsent gewesen sei. Dies wiederum widerspricht dem von französischen Historikern vertretenen Standpunkt, daß es sich beim Faschismus um ein marginales Phänomen gehandelt habe, das vorwiegend auf linke Renegaten und auf einige Intellektuelle eine gewisse Anziehungskraft ausgeübt habe ${ }^{37}$.

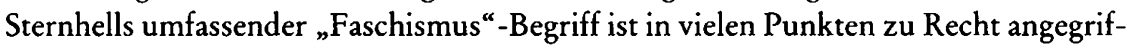
fen worden. Bereits seine außerordentliche Unschärfe macht ihn für eine Auseinandersetzung wenig brauchbar. Seine Definition schließt nahezu alles ein, was antiliberal und nicht zugleich marxistisch ist. Zudem ignoriert er den Charakter des „Faschismus“ als

${ }^{34}$ Sternhell/Sznajder/Ashéri, Naissance, S. 53ff.; Sternhell, Ni droite, S. 35.

${ }^{35}$ Sternhell, Droite révolutionnaire, S. $318 \mathrm{ff}$; ders., Ni droite, S. $136 \mathrm{ff}$.

${ }^{36}$ Rémond, Droites, S. 206ff. Einen guten Überblick über die Forschung geben folgende Arbeiten: Milza, Fascisme Français, S. 11ff.; Dictionnaire, S. 25ff.; Burrin, Le fascisme, S. 603-652. Einen zusätzlichen „Reiz“ erhält die Debatte dadurch, daß Sternhell selbst aus der Schule dieser französischen Historiker stammt, die sich um das Institut d'Etudes Politiques gruppiert. Seine Dissertation über Barrès ist dort unter der Leitung von Raoul Girardet entstanden.

${ }^{37}$ Raoul Girardet spricht von einer „imprégnation fasciste“ einiger Intellektueller wie Pierre Drieu la Rochelle, Robert Brasillach und Lucien Rebatet. Er sieht jedoch den Faschismus nicht in einer inhaltlichen Wandlung der Protagonisten, sondern in dem äußeren Klima, das durch den Aufstieg der diktatorischen Regime in Italien und Deutschland bestimmt war und das eine geistige Anziehungskraft auf die Intellektuellen ausübte; Girardet, Notes, S. 529-546, Zitat S. 530; Serge Berstein folgt in seiner Darstellung dieser Sichtweise: Berstein, La France des années 30, S. $97 \mathrm{f}$. Auch Pierre Marie Dioudonnat betrachtet in seiner Untersuchung der Zeitschrift Je suis partout den Faschismus als eine italienische und deutsche Erscheinung, die ihre Anziehungskraft vor allem auf einige jüngere Intellektuelle aus dem Umkreis der Action française ausübte: Dioudonnat, Je suis partout. Pierre Milza bestreitet entschieden den faschistischen Charakter des Nonkonformismus: Milza, Fascisme Français, S. 207. 
soziale Bewegung und die politischen und wirtschaftlichen Umstände seiner Entfaltung. Er überschätzt damit auch den geistigen Einfluß und die Breitenwirkung der Ideen insbesondere vor dem Ersten Weltkrieg. Schließlich vernachlässigt er die Wandlung der Rahmenbedingungen und damit den Prozeß der Radikalisierung, der die Entwicklung der dreißiger Jahre prägte. Er reiht Belege vom Beginn und Ende des Jahrzehnts aneinander, ohne der Tatsache Rechnung zu tragen, daß die Protagonisten in der Zwischenzeit ihre Position entscheidend verändert haben ${ }^{38}$.

Trotz dieser zutreffenden Vorwürfe haben ihn seine Kritiker in dem entscheidenden Punkt nicht widerlegen können. Sternhell hat die Entstehung einer neuen Rechten nachgewiesen, die in ebenso radikaler Weise wie die extreme Linke die bürgerlich-liberale Mitte bekämpfte ${ }^{39}$. Gerade hier weist der Ansatz von Sternhell grundsätzliche Parallelen zu den Forschungen zur Konservativen Revolution auf. Bereits Armin Mohler hat in einer ausführlichen Besprechung des Werkes $N i$ droite, ni gauche auf diese Übereinstimmungen hingewiesen ${ }^{40}$. Breuer und Sieferle haben mit Blick auf die deutschen Verhältnisse denselben Ausgangspunkt in der Krise des Liberalismus diagnostiziert. Breuers Definition eines neuen Nationalismus läßt sich ohne Einschränkungen auf das von Sternhell beschriebene Phänomen der neuen Rechten anwenden. Selbst das triadische Modell von Sieferle ist weitgehend identisch mit dem Bild, das Sternhell von der Situation in Frankreich entwickelt - mit einem Unterschied: Die Stelle der „Konservativen Revolution“ wird vom "Faschismus" eingenommen. Sowohl Sternhell als auch Sieferle weisen auf die Ähnlichkeit der neuen radikalen Rechten und Linken hin ${ }^{41}$.

In einem Punkt ist jedoch eine Einschränkung angebracht: Sieferle betrachtet, wie bereits erwähnt, auch den Nationalsozialismus als ,reale Konservative Revolution“42. Diese Definition übergeht einen fundamentalen Unterschied zwischen der Konservativen Revolution und dem völkisch-nationalsozialistischen Denken, auf den Breuer hingewiesen hat. In seiner Analyse des Rassegedankens kommt er zu dem Ergebnis, daß derlei Ressentiments besonders bei Ernst Jünger, Wilhelm Stapel, Arthur Moeller van den Bruck und Ernst Niekisch zwar wesentlich weiter verbreitet waren, als dies bisher wahrgenommen wurde. Jedoch schränkt er ein, daß keiner von ihnen die Schwelle zu einer biologistischen Rassenlehre überschritten habe. Das Volk war aus der Sicht der von Breuer untersuchten Konservativen Revolutionäre keine gegebene Größe, keine in der Erbsubstanz determinierte Kategorie, sondern es existierte allenfalls als ein Ideal, das durch andere Prinzipien wie den Staat oder die Nation geformt werden mußte ${ }^{43}$. Auch Sternhell grenzt den Nationalsozialismus von der "faschistischen Ideologie“ ab. Zwar spielt der Antisemitismus in den von ihm beschriebenen Bewegungen ebenfalls eine wichtige Rolle, doch sieht er in ihm nur einen abgeleiteten Aspekt einer umfassenden Gegenbewegung zum Liberalis-

${ }^{38}$ Berstein, La France, S. 83-94; Winock, Fascisme, S. 35-44; Julliard, Sur un fascisme imaginaire, S. 849-861; vgl. Wohl, French Fascism, S. 91-98.

${ }^{39}$ Diese Ansicht unterstützt auch Burrin, Fascisme, S. 630 gegen Rémond, Droites, S. 44f.

${ }^{40} \mathrm{Vgl}$. die ausführliche Besprechung Mohlers von Sternhells Werk Ni droite, ni gauche: Mohler, Konservative Revolution, Bd.2, S. 103ff.; vgl. ders./Mudry/Steukers, Généalogie.

${ }^{41}$ Breuer, Anatomie, S. 195ff.; Sieferle, Konservative Revolution, S. 19ff., 44. Sieferle weist sogar ausdrücklich darauf hin, daß er den Faschismusbegriff wegen seines pejorativen Charakters durch denjenigen der Konservativen Revolution ersetzen möchte.

${ }^{42}$ Sieferle, Konservative Revolution, S. 24.

${ }^{43}$ Breuer, Anatomie, S. $78 \mathrm{ff} ., 95$. 
mus. Der biologische Determinismus des nationalsozialistischen Rassegedankens hingegen begründete eine vollständig neue Ordnung sui generis ${ }^{44}$.

Dieser Unterschied verdient auch deshalb Beachtung, weil so die Einzigartigkeit des nationalsozialistischen Vernichtungswillens ideologisch faßbar wird. In der Vorstellung von unveränderlichen, erblich festgelegten Eigenschaften von Völkern hat der Rassismus seine radikalste Form angenommen. Erst auf dem Boden dieser Lehre ist der Gedanke des Genozid zur furchtbaren Realität geworden. Daher halte ich es ebenso wie Breuer und Sternhell für sinnvoll, zwischen dem biologisch begründeten Rassengedanken und den übrigen Formen des Rassismus und Antisemitismus zu unterscheiden. Hierbei handelt es sich um einen Versuch, das jeweils Typische der beiden Phänomene hervorzuheben. Keineswegs sollen damit die Verstrickungen zahlreicher Konservativer Revolutionäre in die Verbrechen des Dritten Reiches in irgendeiner Form entschuldigt werden.

Noch eine weitere Differenzierung scheint mir notwendig zu sein. Damit der Begriff des Faschismus durch seinen inflationären Gebrauch nicht jegliche analytische Kraft verliert, sollte er deutlich von der Konservativen Revolution und dem Nonkonformismus abgrenzbar sein. Die Herkunft des Wortes aus dem Arsenal der politischen Kampfbegriffe hat es ohnehin außerordentlich erschwert, die Auseinandersetzungen auf einer sachlichen Ebene zu führen. Dies hat entscheidend zur Verhärtung der Fronten zwischen Sternhell und den französischen Historikern beigetragen und eine differenzierte Betrachtung weitgehend verhindert. Der Blick auf die Ideologien reicht allerdings nicht aus, um festzustellen, ob die jeweiligen Bewegungen als faschistisch bezeichnet werden müssen. Vielmehr muß sich die Definition auf einen generischen Begriff stützen, der ein Minimum an Übereinstimmung mit dem italienischen „Original“ aufweist. Dazu gehört in erster Linie eine Massenbewegung mit paramilitärischer Organisation und autoritärer Führungsstruktur ${ }^{45}$.

Es wird niemand bestreiten, daß der Faschismus auch eine transnationale Dimension aufweist, die in der Nachahmung des italienischen Vorbildes deutlich wird. Allerdings läßt er sich nicht darauf reduzieren. In erster Linie muß er als nationalistische Erscheinung und damit als Antwort auf eine spezifisch nationale Krise betrachtet werden. Die amerikanischen Historiker William D. Irvine und Robert Soucy haben in ihren Untersuchungen deutlich gemacht, daß die französische Rechte keineswegs resistent gegenüber radikal antidemokratischen und militaristischen Einflüssen war. Im Unterschied zur französischen Historiographie sind sie der Ansicht, daß der französische Faschismus in der plebiszitär-bonapartistischen Tradition der Rechten seine Wurzeln hat, und betrachten daher die Ligen als Keimzellen des Phänomens ${ }^{46}$. Die Radikalität des Faschismus zeigt sich in der Wahl seines Gegners. Weitgehend besteht Einigkeit darüber, daß die

${ }^{44}$ Sternhell, Roots, S. 133.

$45 \mathrm{Vgl}$. zu den Kriterien Burrin, Le fascisme, S. 620ff. Bei diesen Ausführungen stütze ich mich auch auf die Überlegungen, die Andreas Wirsching im Graduiertenkolleg Trier am 24.6. 1994 vorgetragen hat: Französischer „Faschismus“ in der Zwischenkriegszeit. Empirische Befunde und vergleichende Perspektiven.

46 Soucy sieht wie Sternhell die geistigen Wurzeln des Faschismus in Frankreich im ausgehenden 19. Jahrhundert; vgl. Soucy, First Wave, S. 1ff.; ders., Second Wave; ders., Das Wesen des Faschismus in Frankreich, S. 46-85; Irvine, Fascism in France, S. 271-295; vgl. Wippermann, Europäischer Faschismus, S. 198. 
Hauptfeindschaft dem Kommunismus galt, den er durch die Ausbildung einer radikal entgegengesetzten, aber doch verwandten Ideologie zu vernichten trachtete und diese Absicht auch in die Tat umsetzte ${ }^{47}$.

Von diesen Bewegungen unterscheiden sich die Nonkonformisten vor allem durch ihre Organisationsstruktur. Es handelte sich meist um kleinere Zirkel, die sich um eine Zeitschrift gruppierten und ihren Einfluß vorwiegend über ihre publizistische Tätigkeit geltend machten. Wenn sie als faschistisch zu bezeichnen sind, so vor allem deshalb, weil sie Anhänger, Sympathisanten oder Ideenlieferanten französischer Bewegungen waren, die diese Kriterien erfüllt haben.

Weiterhin muß die Radikalität der Ideen überprüft werden. In welcher Form und bis zu welchem Grad hielten die Nonkonformisten Gewalt für legitim? Dies wird insbesondere in den Stellungnahmen zu konkreten Ereignissen sichtbar. Wichtige Hinweise liefert auch hier der Adressat der jeweiligen Attacken. Richteten sie sich primär gegen das bürgerlich-liberale System, dessen Herrschaft bereits im Niedergang begriffen war, oder gegen eine kämpferische Arbeiterbewegung, der man mit ähnlichen Kampfmethoden entgegentreten wollte?

Als Ergebnis dieser ersten Eingrenzung läßt sich festhalten: Die Ansätze zur Konservativen Revolution und zum Nonkonformismus lassen sich auf einen vorläufigen gemeinsamen Nenner bringen: Die Intellektuellen waren Protagonisten eines neuen Nationalismus, der durch eine doppelte Feindschaft zum Liberalismus und zum Sozialismus gekennzeichnet war. Die größeren Gemeinsamkeiten hatten sie mit dem Gegner auf der Linken. Mit ihm verband sie der jakobinische Charakter ihrer Ideologie, die revolutionäre Dynamik und schließlich der Appell an ein kollektives Ideal. An die Stelle der "Klasse" trat jedoch die "Nation". Von den Nationalsozialisten unterschieden sie sich dadurch, daß die „Rasse“ kein zentrales Kriterium ihrer Ideologie bildete. Von den Anhängern eines Faschismus wiederum lassen sie sich durch einen geringeren Grad der Radikalisierung abgrenzen.

Im folgenden Kapitel werden die Rahmenbedingungen des Vergleichs genauer abgesteckt: die unterschiedlichen Phasen der Kritik und ihre politischen Hintergründe, die Sozialisation der Protagonisten und ihre Generationserfahrungen gerade im Hinblick auf das Kriegserlebnis sowie die Besonderheiten der politischen Kulturen (Kapitel I). Die weiteren Kapitel konzentrieren sich auf die Intellektuellenzirkel der Jeune Droite und des Ordre Nouveau. Die beiden Gruppierungen bildeten zusammen mit dem Esprit-Kreis um Emmanuel Mounier den Kern des Nonkonformismus, den Touchard, Loubet del Bayle und Sternhell beschrieben haben. Da für die Gruppe um Esprit durch die beiden Monographien von Michel Winock ${ }^{48}$ und John Hellman ${ }^{49}$ bereits eine quellennahe Darstellung vorliegt, habe ich auf eine nochmalige Behandlung verzichtet. Allerdings sind aufgrund der zeitweise engen Verbindungen zwischen dem Ordre Nouveau und Esprit Seitenblicke unerläßlich.

Die Gegenüberstellung von Jeune Droite und Ordre Nouveau ist von besonderem Interesse, weil sie von unterschiedlichen Ausgangspunkten zu ähnlichen Positionen ge-

\footnotetext{
${ }^{47}$ Rémond, Droites, S. 201; Soucy, First Wave, S. XIX; Burrin, Fascisme, S. 623f.; Nolte, Faschismus, S. 51.

48 Winock, Esprit.

${ }^{49}$ Hellman, Mounier.
} 
langten und sich einander annäherten. Die Vertreter der Jeune Droite stammten aus dem Umfeld der gegenrevolutionären Action française, während zwei führende Mitarbeiter des Ordre Nouveau, Robert Aron und Arnaud Dandieu, mit sozialistischen Ideen sympathisierten. So wird die Geschichte ihrer Annäherung zur Geschichte der Revision ihrer politischen Standpunkte (Kapitel II).

Die weitere Darstellung orientiert sich am Gang der politischen Ereignisse. In erster Linie sollen damit Entwicklungen, Wandlungen und Brüche hervorgehoben werden, die aus der Perspektive von Sternhell ausgeblendet wurden. Eine solche Lokalisierung der Äußerungen im politischen Kontext über das gesamte Jahrzehnt hin fehlt bisher auch in den übrigen Untersuchungen zu den Nonkonformisten. Doch nur so wird die Entstehung der Ideen in ihrem Kommunikationszusammenhang und den jeweiligen politischen Frontstellungen sichtbar.

Den Auftakt bildet die Außenpolitik. Eine zentrale Rolle spielten die Radikalisierung des deutschen Revisionismus und der Aufstieg des Dritten Reiches. Die Wahrnehmung des Nachbarn ist stark bestimmt durch die Rezeption der Schriften der Konservativen Revolution und durch den Aufbau persönlicher Beziehungen. Die Rolle Deutschlands für die spezifische Argumentationshaltung der Nonkonformisten ist bisher kaum ausreichend beleuchtet worden (Kapitel III).

Zwei Zäsuren gliedern die Untersuchung: Die eine markiert der Aufmarsch der Ligen vor dem Sitz der Nationalversammlung im Februar 1934. Diese Demonstration löste eine Debatte um die Existenz eines französischen Faschismus aus und schuf damit ein neues politisches Klima, das sich auf die Strategieentwürfe und Bündnisse der beiden Gruppierungen auswirkte. Ein Großteil der französischen Forschung geht mehr oder weniger stillschweigend davon aus, daß diese Zäsur bereits das Ende des Nonkonformismus in Frankreich einläutete, der zwischen den neuen Fronten von Faschismus und Antifaschismus aufgerieben wurde. Diese Ansicht wird durch die Analyse der Argumentationsfiguren und die Rekonstruktion der Verflechtungen, die sich auf neue Quellenfunde stützen, widerlegt (Kapitel IV).

Die zweite Zäsur läßt sich an der Auseinandersetzung über den Krieg Italiens in Abessinien im Oktober 1935 festmachen. Sie leitete eine Phase der innenpolitischen Radikalisierung ein, die von der ideologischen Konfrontation zwischen Kommunismus einerseits und Nationalsozialismus und Faschismus andererseits überlagert wurde. Sie muß als Phase der Entscheidung betrachtet werden, in der die ideologischen Weichen für die Zeit der Besatzung, für Widerstand, Defaitismus und Kollaboration gestellt wurden. Gerade in dieser extremen Situation werden grundlegende Denkmuster der nonkonformistischen Gruppierungen sichtbar (Kapitel V).

Zum Schluß werde ich die vergleichende Fragestellung wieder aufgreifen. Der Begriff der Konservativen Revolution wird anhand der Ergebnisse auf seine Tragfähigkeit hin überprüft und modifiziert. Schließlich werden die Perspektiven für die Forschung zum Phänomen der neuen Rechten in Frankreich und Deutschland skizziert.

Die wichtigste Quellengruppe bilden die Veröffentlichungen der Nonkonformisten: ihre Werke und die Artikel, die sie in eigenen und fremden Zeitschriften veröffentlicht haben. Sie geben wertvollen Aufschluß über die Aktivitäten in benachbarten Gruppierungen und die wechselseitigen Verflechtungen, die bisher nicht ausreichend berücksichtigt wurden. Bei der Rekonstruktion der Kommunikationsbeziehungen ist die Memoirenliteratur schon allein wegen ihres Umfangs eine bedeutende Quelle. Sie liefert 
wertvolle Informationen über Bekanntschaften, Beziehungen und Affinitäten. Bei aller gebotenen Vorsicht aufgrund möglicher Rechtfertigungsabsichten lassen sich so persönliche Kontakte und biographische Hintergründe rekonstruieren. Ergänzend dazu hatte ich 1994 die Gelegenheit zu einem Gespräch mit Alexandre Marc, der als Mitgründer und Motor vom Ordre Nouveau mir einige wertvolle Einblicke in die nonkonformistischen Kreise geben konnte.

Einen archivalischen Kernbestand gibt es hingegen nicht. Spuren finden sich an den verschiedensten Stellen: in Polizeiberichten, Nachlässen, Botschafts- und Universitätsarchiven. Wertvolle Hinweise liefern insbesondere die Polizeiberichte, die im Pariser Nationalarchiv und in der Polizeipräfektur liegen und die zu diesem Thema bisher nicht ausgewertet wurden. Sie sind vor allem für die Jeune Droite ergiebig. Wichtige Quellen über die Kontakte nach Deutschland befinden sich im Politischen Archiv des Auswärtigen Amtes. Ergänzende Informationen können den Beständen des Bundesarchivs Koblenz (Plans-Kongreß) und dessen Außenstelle Berlin-Zehlendorf (Abetz) sowie den Beständen des Staatsarchivs Würzburg (Reichsstudentenführung/Thierry Maulnier) und des Universitätsarchivs Frankfurt (Denis de Rougemont) entnommen werden. Im Institut für Zeitgeschichte in München befinden sich die Briefe von Harro Schulze-Boysen an seine Eltern, die weitere Einblicke in die Beziehungen zwischen dem Ordre Nouveau und dem Gegnerkreis geben. Diese Quellen sind zum Teil bereits unter anderen Fragestellungen ausgewertet worden; sie liefern jedoch gerade für die Frage der Beziehungen und Affinitäten zwischen Konservativer Revolution und Nonkonformismus neue Einblicke und Sichtweisen.

Andere Archivbestände geben Aufschluß über die interne Organisation und die Aktivitäten des Ordre Nouveau: der Nachlaß von Arnaud Dandieu und die Briefe von Gabriel Marcel in der Pariser Nationalbibliothek, sowie der Nachlaß von Denis de Rougemont in Neuchâtel in der Schweiz. Dandieus Nachlaß enthält zwar eine Fülle von philosophischen Entwürfen und Artikeln, die ein deutliches Licht auf die Herkunft mancher Ideen werfen, ist jedoch für die Entstehungsgeschichte der Gruppierung wenig ergiebig, da Dandieu bereits 1933 starb. Die Briefe Marcels helfen dagegen, einige Auseinandersetzungen innerhalb des Ordre Nouveau durch neue Einsichten zu ergänzen. Der Rougemont-Nachlaß ist bereits von dessen Biograph Bruno Ackermann ausgewertet worden, er liefert darüber hinaus jedoch weitere wertvolle Informationen zur Entwicklung vom Ordre Nouveau und dem Umfeld der Gruppierung.

Insgesamt gleicht die Rekonstruktion der personellen Verflechtungen und die Einbettung in den politischen Diskussionszusammenhang der 30er Jahre der Arbeit an einem Puzzle, bei dem die meisten Teile verlorengegangen und die übrigen verstreut sind. In ihren Einzelaussagen sind die archivalischen Quellen häufig fragmentarisch, erst die Verknüpfung der verschiedenen Informationen untereinander und mit den gedruckten Äußerungen liefert neue und teilweise überraschende Ergebnisse.

Eine Anmerkung zur Schreibweise: Kursiv werden Titel von Büchern und Zeitschriften geschrieben, ohne Auszeichnung erscheinen hingegen Bewegungen, Gruppierungen und Institutionen. So lassen sich Bewegungen und Zeitschriften mit dem gleichen $\mathrm{Na-}$ men unterscheiden: etwa die Bewegung Action française und ihre Zeitung Action française, die Gruppierung Ordre Nouveau und ihre Zeitschrift Ordre Nouveau. 